\title{
Bronchopulmonary Dysplasia Prediction Using Support Vector Machine and LIBSVM
}

\author{
Marcin Ochab \\ AGH University of Science and Technology, \\ 30 Mickiewicza 30-059 Kraków, Poland \\ Email: marcin.ochab@labor.it.pl
}

\author{
Wiesław Wajs \\ AGH University of Science and Technology, \\ 30 Mickiewicza 30-059 Kraków, Poland \\ Email: wwa@agh.edu.pl
}

\begin{abstract}
The paper presents BPD (Bronchopulmonary Dysplasia) prediction for extremely premature infants after their first week of life. SVM (Support Vector Machine) algorithm implemented in LIBSVM[1] was used as classifier. Results are compared to others gathered in previous work [2] where LR (Logit Regression) and Matlab environment SVM implementation were used. Fourteen different risk factor parameters were considered and due to the high computational complexity only 3375 random combinations were analysed. Classifier based on eight feature model provides the highest accuracy which was 82.60\%. The most promising 5 -feature model which gathered 82.23\% was reasonably immune to random data changes and consistent with $L R$ results. The main conclusion is that unlike Matlab SVM[2] implementation, LIBSVM can be successfully used in considered problem, but it is less stable than LR. In addition, the article discusses influence of the model parameters selection on prediction quality.
\end{abstract}

\section{INTRODUCTION}

B RONCHOPULMONARY dysplasia (BPD) is a chronic pulmonary morbidity which affects premature infants [3], [4]. It is most common among children who received prolonged mechanical ventilation to treat respiratory distress syndrome [5], [6] and those with low birth weight. Almost a third of infants with birth weight lower than 1000g [7] are affected. Due to the fact that the disease is poorly understood, many projects are focused on identifying its factors of risk. Since it can not be diagnosed until a 28th day of life [8], it is very important to predict such a result after the end of the first week, which would enable an early prevention of the disease[9]. Therefore, an intensive work has been done to define a classifier, based on static parameters (gathered after birth) and dynamic ones (collected during the first week of life), which would be able to predict the diagnosis. Although several prediction models of BPD [10], [11], [12], [13], [14], [15], [16], [17], [18], [19] used in research have been reported, none of them could be used in common clinical practice due to the variety of reasons and none use SVM.

\section{RELATED WORKS}

As mentioned before, there are numerous works related to BPD, its risk factors and prediction [20], [21], [22], [23]. The most popular one is the analysis of static data whose main features are gestational age and birth weight. The other factors considered are admission of surfactant, presence of patent ductus arteriosis $(P D A)$, or respiratory support. In addition, dynamical data (which is much harder to obtain) is analysed in more sophisticated models. Most of such parameters are: arterial blood gas variables like fraction of inspired oxygen $\left(\mathrm{FiO}_{2}\right)$ or alveolar-arterial ratio $(\mathrm{AA})$ [24] (which is respiratory distress degree measure); blood gas levels like oxygen saturation of arterial hemoglobin $\left(\mathrm{SpO}_{2}\right)$ and its standard deviation, mean value etc. [25] or even time series analysis [26]; heart beat and its derivatives.

$$
A A=\frac{p O_{2}}{p_{A T M} \cdot \mathrm{FiO}_{2}-p C \mathrm{O}_{2}},
$$

where $\mathrm{pO}_{2}$ - oxygen partial pressure, $p_{A T M}$-atmospheric pressure, $\mathrm{pCO}_{2}$ - carbon dioxide partial pressure, $\mathrm{FiO}_{2}-$ fraction of inspired oxygen.

Some of the papers introduced race and ethnicity or sex as factors which seem to be promising but require a very big set of data. It should be indicated that the vast majority of studies uses logit regression (LR) in prediction. Best LR models gain about $73 \%$ to $82 \%$ of accuracy. Many of authors mention use of support vector machine(SVM)[27] in future works, however it is difficult to find them. In our previous work[2] we compared SVM with LR classifiers. Unfortunately, due to internal Matlab SVM library usage results were highly unsatisfactory. The highest accuracy gained was only $79.39 \%$. Moreover, the bigger features set was used the worse results we got. In general, only three and four feature models were able to gain accuracy higher than $70 \%$. That is why we decided to use LIBSVM implementation instead, which gave us a very wide scope of parameters tuning. Although additional parameters highly increased computational complexity of optimal model search, even limited random parameters space exploration gave us quite good results, comparable to LR.

\section{GENERAL IDEAS OF USED METHODS}

\section{A. Logistic regression}

Probability of the dependent variable equalling a BPD positive diagnosis $\left(y_{k}=1\right)$, on condition that explanatory variables (features of specific case $k$ ) equals $X_{k}=\left(x_{1, k}, x_{2, k}, . ., x_{n, k}\right)$, we define as:

$$
p_{k}=P\left(y_{k}=1 \mid X_{k}\right)=\frac{e^{a_{0}+\sum_{i=1}^{n} a_{i} x_{i}}}{1+e^{a_{0}+\sum_{i=1}^{n} a_{i} x_{i}}},
$$




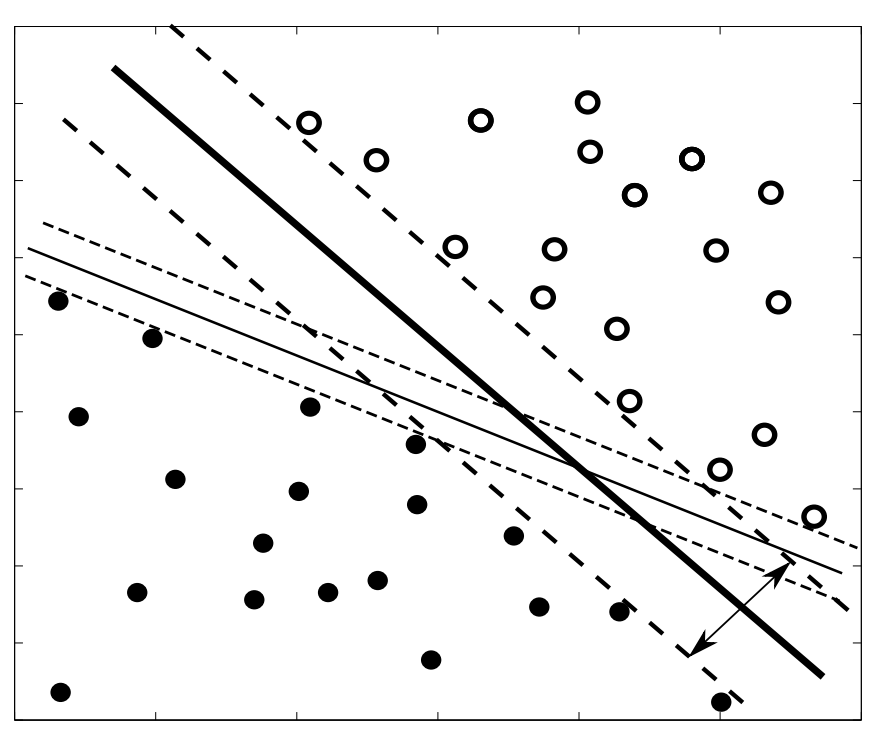

Fig. 1. Maximizing margin in SVM method.

where $x_{i, k}$-explanatory variables (feature values for case $k$ ), $a_{i}$-regression coefficients, $n$-number of features.

In contrast to linear regression, where we assume normal distribution of the independent variables and because explanatory variables variance are not equal, we can not use the method of least squares to obtain regression coefficients. Thus they are usually calculated using maximum likelihood estimation, maximizing likelihood function $(L)$ or minimizing its negative logarithm using learning data:

$$
\begin{gathered}
L=\prod_{y_{k}=1} p_{k} \prod_{y_{k}=0}\left(1-p_{k}\right), \\
\ln (L)=\sum_{k=1}^{m}\left[y_{k} \cdot \ln \left(p_{k}\right)+\left(1-y_{k}\right) \cdot \ln \left(1-p_{k}\right)\right],
\end{gathered}
$$

where $k$-observation number (learning case), $y_{k}$-diagnosis for case $k, m$-number of observations.

Having $a_{i}$ regression coefficients, we can easily predict BPD positive diagnosis probability of case X using Eq. 2.

\section{B. Support vector machine}

We define the learning data $\mathrm{D}$ divided in two classes $\mathrm{y}$ as:

$$
D=\left\{\left(X_{k}, y_{k}\right) \mid X_{k} \in R^{n}, y_{k} \in\{1,-1\}\right\}_{k=1}^{m}
$$

We are looking for hyperplane

$$
W \bullet X+b=0
$$

which separates classes and provides maximum margin as on Fig. 1, which is the same as the problem of minimizing $L$ :

$$
L(W)=\frac{\|W\|^{2}}{2}+c \cdot \sum_{k=1}^{m} \varepsilon_{k},
$$

with conditions:

$$
y_{k}\left(W \cdot \phi\left(X_{k}\right)+b\right) \geq 1-\varepsilon_{k}
$$

where $\varepsilon \geq 0$-slack variable, $c>0$-penalty parameter for each point wrongly classified, $\phi$-kernel function.

Thanks to the kernel functions for non linear separable problems, we can transform original data from $n$ dimensional space to $p$ dimensional ( $p>n$, as on Fig. 2), in which there is much higher likelihood that they will be linear separable.

\section{DAta AND Methods}

Data was collected thanks to the Neonatal Intensive Care Unit of The Department of Pediatrics at Jagiellonian University Medical College using our own software. It includes 109 patients born prematurely with birth weight less than or equal to $1500 \mathrm{~g}$ admitted, no later than on the second day of life. For 46 of them BPD have been diagnosed after fourth week of life.

To build a suitable model 14 different features mentioned in literature were considered:

- Binary such as:

- presence of patent ductus arteriosus $(P D A)$ [28] ,

- use of a respirator $(R E S P I M V)$ during the first week of life,

- administration of surfactant (SURFACT) [29] in the same period.

- Real-Valued (values range in parentheses) such as:

- birth weight (BWEIGHT) (550-1500g),

- gestational age (GAGE) (22-34 weeks),

- alveolar-arterial ratio $(A A)(0.05-1)$ measured during patient admission,

- a percentage of the time during first week for which the oxygen saturation of hemoglobin was less than $85 \%$ ( $L O W 85)(0.03 \%-12.45 \%)$ or higher than $94 \%$ (HIGH94) (14.56\%-99.02\%),

- average number of heartbeats per minute (BPMMEAN)[5](124.69-161.42 bpm),

- mean and standard deviation of oxygen saturation (SPO2MEAN, SPO2DEV) (accordingly $89.89 \%-98.99 \%$ and 1.19-7.98) and their trends (first day to first week ratio: BPMMEAN_TR, SPO2MEAN_TR, SPO2DEV_TR) (accordingly 0.8-1.18, 0.96-1.07 and $0.51-2.3 \overline{6})$.

Accuracy $(A C C)$ defined as below was considered as preliminary result measure. The sensitivity $(T P R)$ and specificity $(S P C)$ were also obtained:

$$
\begin{gathered}
A C C=\frac{T P+T N}{T P+T N+F P+F N}, \\
T P R=\frac{T P}{T P+F N}, \\
S P C=\frac{T N}{T N+F P}
\end{gathered}
$$




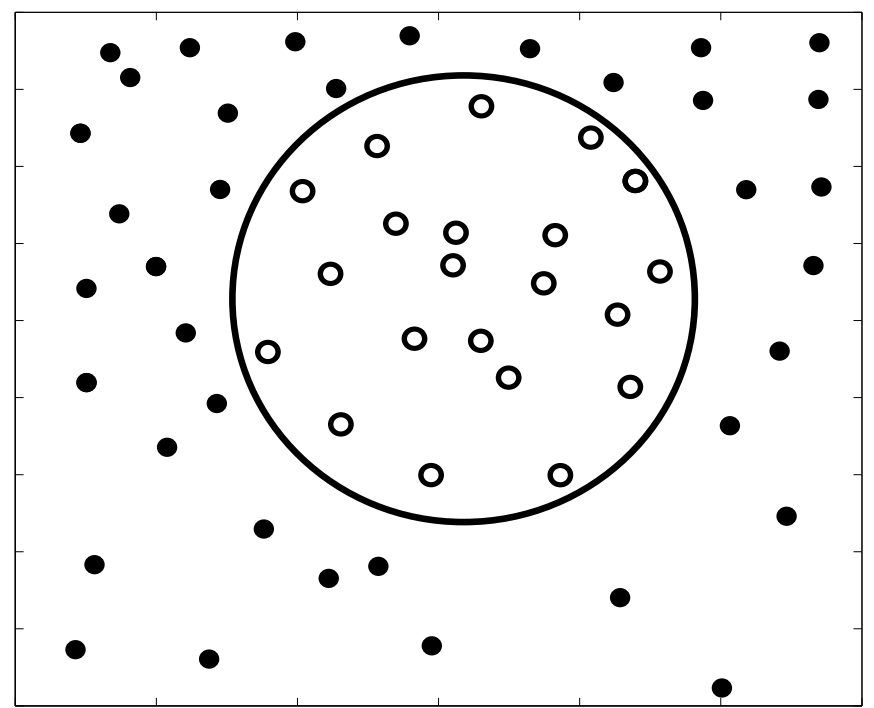

(a) data in $R^{2}$ space

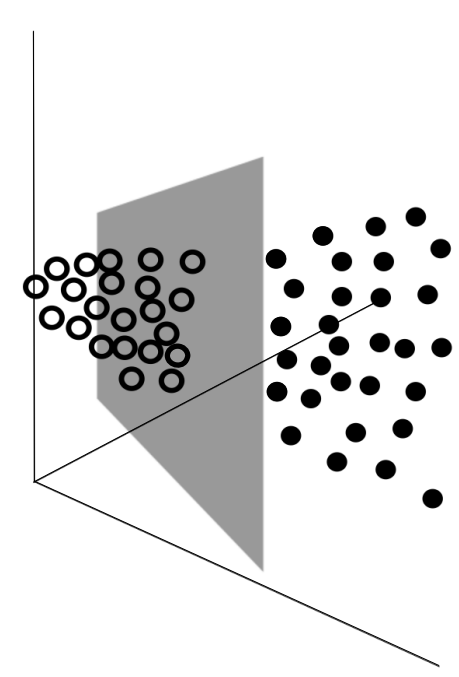

(b) data transformed to $R^{3}$ space

Fig. 2. Making data linear separable with dimensional transformation.

where $T P$-True Positives, $F P$-False Positives, $F N$-False Negatives, $T N$-True Negatives.

As mentioned before in SVM computation version 3.17 of LIBSVM library was used. Based on a few arbitrary chosen and tested models it has been found that for specified problem C-SVC method is more effective than nu-SVC (range of parameter c (Eq. 7) is from zero to infinity, rather than as in nu always between [0,1]). It has also been discovered that sigmoid kernel function gives better results and is much faster in finding the separating hyperplane than radial basis (RBF) one.

$$
\begin{gathered}
K\left(X_{i}, X_{j}\right) \equiv \phi\left(X_{i}\right)^{T} \phi\left(X_{j}\right), \\
R B F: K\left(X_{i}, X_{j}\right)=e^{-\gamma\left\|X_{i}-X_{j}\right\|^{2}}, \gamma>0, \\
\text { Sigmoid: } K\left(X_{i}, X_{j}\right)=\tanh \left(\gamma X_{i}^{T} X_{j}+r\right),
\end{gathered}
$$

where $\gamma, r$-kernel parameters.

As suggested in library documentation, data have been normalized to $[-1,1]$ range and optimization of parameters $\gamma\left(\gamma=2^{-15}, 2^{-14}, \ldots, 2^{3}\right)$ and $c\left(c=2^{-5}, 2^{-4}, \ldots, 2^{15}\right)$ was performed for each model (Fig. 3). Unlike suggested, in presented results we did not use grid.py script which provides cross-validation and parameters optimization. This method gives very promising results for considered problem, achieving easily up to $83-86 \%$ of $A C C$, but models found with this method turned out to be very unstable. Any random change of data (removing or adding samples) significantly decreased its accuracy.

It is very important to find a model the most possibly independent on specific learning data. Therefore, we decided to use a method similar to Jacknife [30]: for each pair $c$ and $\gamma$ parameters calculations were repeated 30 times, each time randomly excluding 30 samples of data and using crossvalidation procedure (each patient was treated as a test sample while all other data was learning set) on the rest of it. This way deviation and mean value of accuracy, sensitivity and specificity were obtained, which gives an estimate on the model 'sensitivity' to data structure (it might be important when calculating on such a little data set as 109 patients). It should be mentioned that each time we refer in this paper to $A C C, T P R$ or $S P C$ values we mean average computed as above. The test was repeated once again for the best results, excluding the data of only 10 random patients - it shows wether extension of learning data increases or decreases accuracy and overfitting occurs. Due to the high computational complexity of proposed optimisation procedure only 3375 random models containing 2 to 14 parameters were analyzed - it took more than a week for modern 8-core Intel Core i7 based PC. Nevertheless, such little number of experiments gave satisfactory results.

To compare with LR algorithm we used data from previous article[2], where we reviewed all of the $2^{14}$ possible combinations of models with exactly same Jacknife and crossvalidation procedure.

\section{RESUlTS}

The most essential results are presented in Table II . To compare, in each presented model mean value of $A C C, T P R$ and $S P C$ were obtained with different Jacknife parameters, using both methods: LR and sigmoid SVM with LIBSVM. Where applicable we added RBF Matlab SVM implementation results (as M. SVM) from [2].

According to the assumptions in previous section the highest mean value of accuracy among SVM results gained eightparameter model with $82.60 \%$. Unfortunately standard deviation of $A C C$ was 5.15 , which in contrast to others was 
TABLE I

SVM MODELS WITH BOTH TPR AND SPC HIGHER THAN $80 \%$

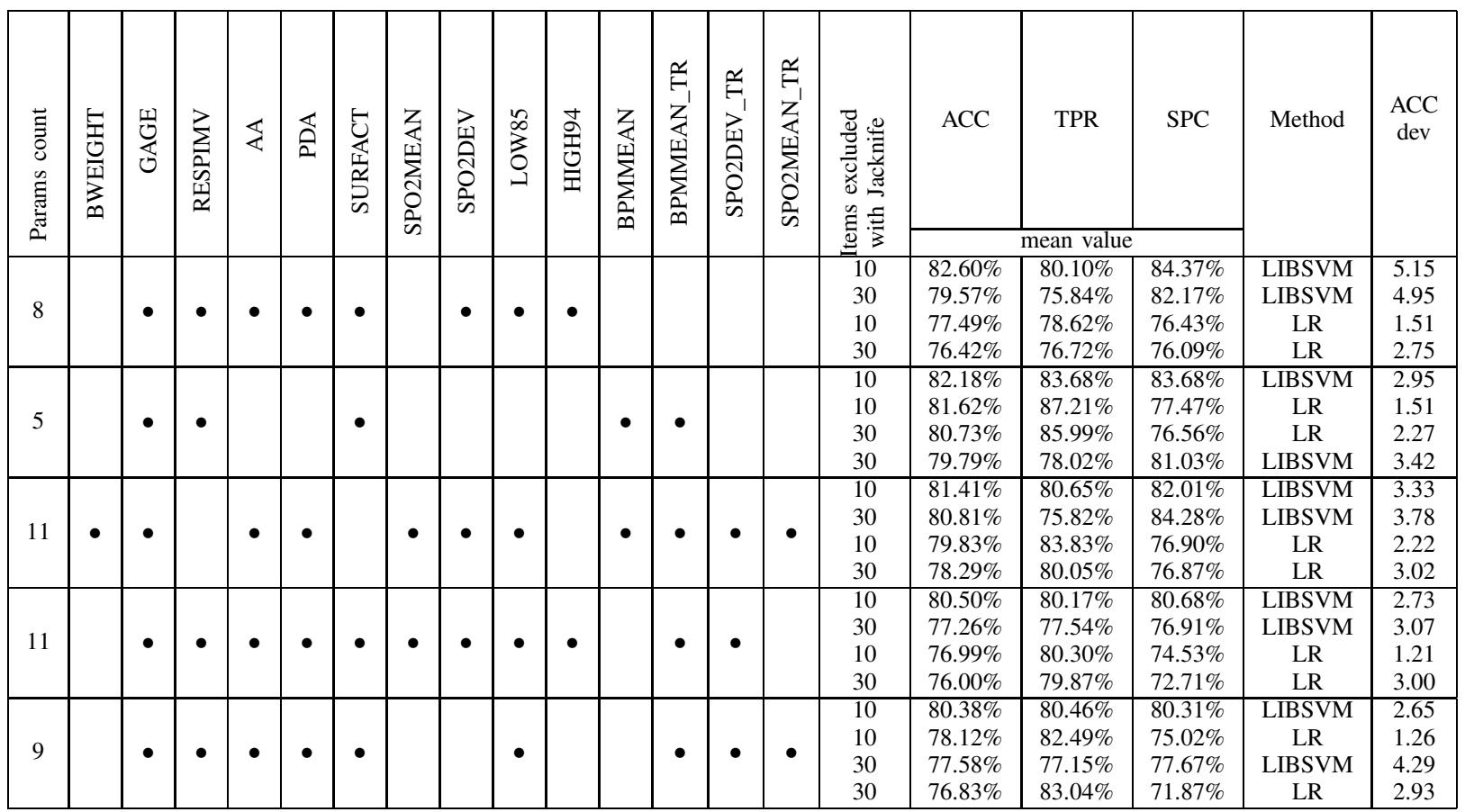

very high (STD values are presented in Table III). The TPR value was $80.10 \%$ and $S P C 84.37 \%$, which is a very good result in comparison with the fact that only five of examined SVM models achieved both parameters higher than $80 \%$ (see Table I).

Next we present generally the best $A C C$ model which was six-parameter LR which gained $82.79 \%$ with $T P R=84.20 \%$, $S P C=81.73 \%$ and standard deviation of $A C C$ as low as 1.11 . Both above models give quite good results, but only using specific algorithm (LR or SVM).

In contrast to the above fact, we present next two best five- and six-parameter models which give accuracy higher than $81 \%$ for both methods. Particularly noteworthy model is the five-parameter one - it gives respectively $82.23 \%$ for SVM and $82.59 \%$ for LR of $A C C$ which was the best of examined five-parameter models for both methods. Although the TPR result for SVM was only $77.13 \%$, this model seems to be a very reasonsonable choice with $\mathrm{SPC}=85.87 \%$ for $\mathrm{SVM}$ and $T P R=84.65 \%, S P C=81.07 \%$ for LR. Even in that case, where standard deviation of $A C C$ for SVM was 3.04, it was about twice higher than 1.49 for LR. An interesting similar 5-parameter model with little lower accuracy is presented in Table I.

Afterwards we made a review of the best accuracy results for each method and each model size:

- 4-parameter models - for LR we succeeded to obtain $82.01 \%$ of $A C C$ and only $80.85 \%$ for SVM. However, this second model (bolded in Table III) draws attention due to its simplicity, low $A C C$ standard deviation (about 1.4) and $A C C$ higher than $80 \%$ for both methods.

- 3-parameter models - $81.29 \%$ for LR and $80.57 \%$ for $\mathrm{SVM}$ are good results just as standard deviation of $A C C$ lower than 1.5, though none of models exceeds the psychological barrier of $80 \%$ of $A C C$ for both methods at a time.

- 2-parameter models - SVM results are unsatisfactory due to $76.01 \%$ of highest $A C C$ and its standard deviation as high as 5.81 . However, LR was able to gain $80.30 \%$ of $A C C$, which is quite interesting.

Lastly, it must be noted that full 14-parameter SVM model gained $76.86 \%$ and LR $77.55 \%$ of $A C C$. As a graphic example two-parameter model result was presented on Fig. 4.

\section{DISCUSSION}

The first conclusion is that SVM classification algorithm can be almost as accurate as LR and if its parameters are properly chosen it gives rewarding results, even for a complicated multiparameter model of BPD. The best choice for such a prediction is to use the LIBSVM instead of Matlab's implementation, which gives less control on computation process. Most likely that was the reason why the bigger parameter set we used the worse results we got using Matlab[2]. Although we did not test all possible $2^{14}$ combinations of parameters (only 3375 random models), nonetheless looking on Table III it can be concluded that standard deviation of accuracy for SVM is much higher than for logit regression. Using bigger learning set (only 10 samples excluded) for best results it reaches even 
TABLE II

MODELS AND METHODS ACCURACY COMPARISON

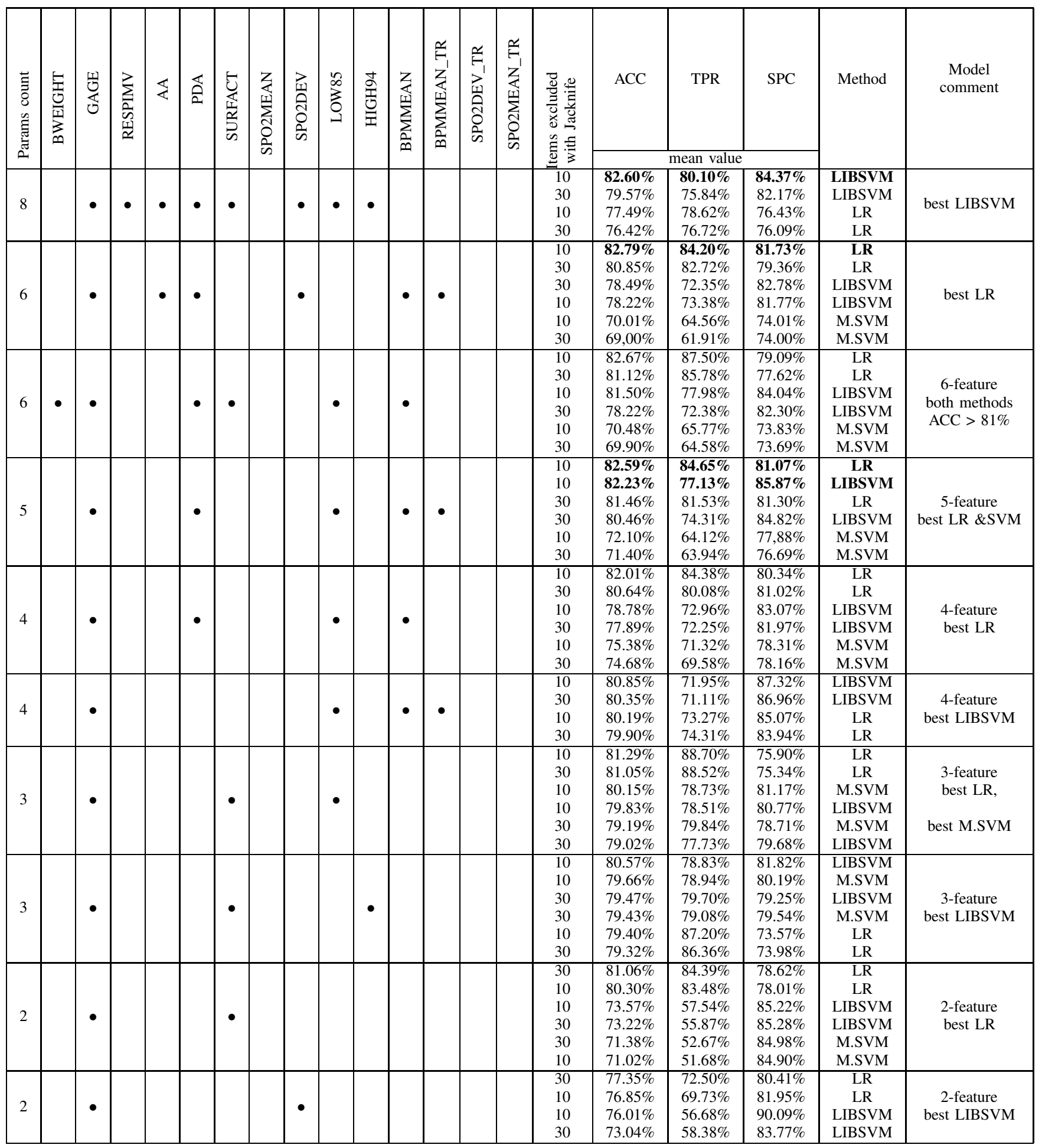




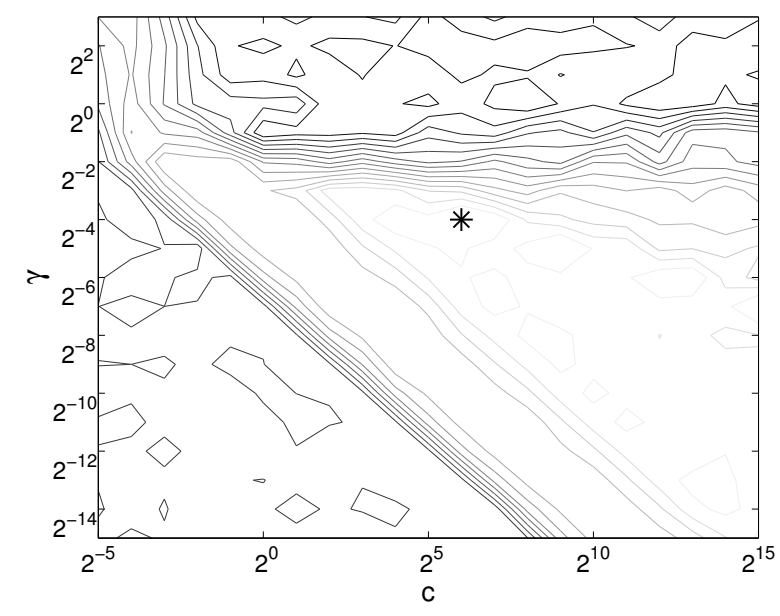

(a) eight-parameter model

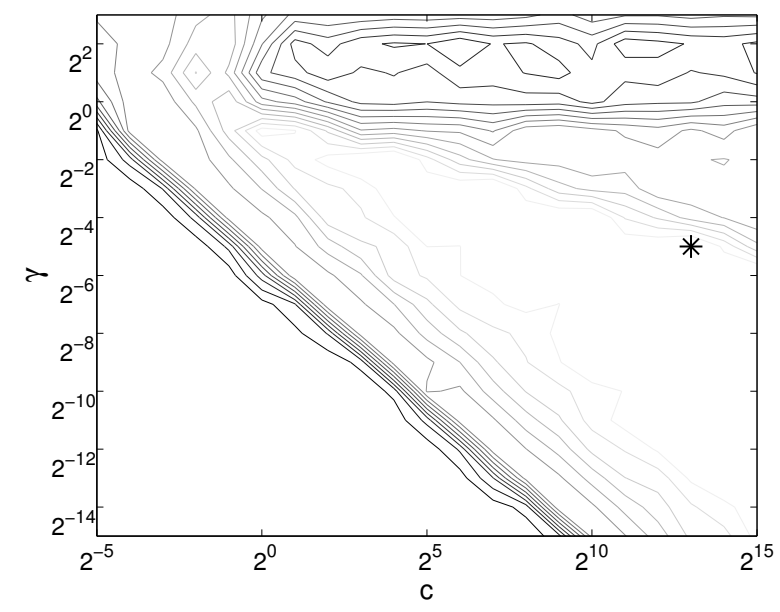

(b) five-parameter model

Fig. 3. Parameter $\gamma$ and $c$ optimization for eight-parameter and five-parameter models bolded in Table II.

TABLE III

STANDARD DEVIATION OF MODELS ACCURACY

\begin{tabular}{|c|c|c|c|c|c|}
\hline \multirow{2}{*}{$\begin{array}{c}\text { Parameters } \\
\text { count }\end{array}$} & \multirow{2}{*}{ Method } & \multicolumn{4}{|c|}{ Jacknife excluded count } \\
\cline { 3 - 6 } & & \multicolumn{2}{|c|}{10} & \multicolumn{2}{|c|}{30} \\
\cline { 3 - 6 } & & ACC & ACC STD & ACC & ACC STD \\
\hline \multirow{2}{*}{8} & LIBSVM & $82.60 \%$ & 5.15 & $79.57 \%$ & 4.95 \\
& LR & $77.49 \%$ & 1.51 & $76.42 \%$ & 2.75 \\
\hline \multirow{2}{*}{6} & LR & $82.79 \%$ & 1.11 & $80.85 \%$ & 2.52 \\
& LIBSVM & $78.22 \%$ & 1.96 & $78.49 \%$ & 2.74 \\
\hline \multirow{2}{*}{6} & LR & $82.67 \%$ & 1.19 & $81.12 \%$ & 2.56 \\
& LIBSVM & $81.50 \%$ & 4.89 & $78.22 \%$ & 5.06 \\
\hline \multirow{2}{*}{5} & LR & $82.59 \%$ & 1.49 & $81.46 \%$ & 2.51 \\
& LIBSVM & $82.23 \%$ & 3.04 & $80.46 \%$ & 2.44 \\
\hline \multirow{2}{*}{4} & LR & $82.01 \%$ & 1.73 & $80.64 \%$ & 2.60 \\
& LIBSVM & $78.78 \%$ & 2.68 & $77.89 \%$ & 4.36 \\
\hline \multirow{2}{*}{4} & LIBSVM & $\mathbf{8 0 . 8 5 \%}$ & $\mathbf{1 . 3 9}$ & $80.35 \%$ & 2.28 \\
& LR & $\mathbf{8 0 . 1 9 \%}$ & $\mathbf{1 . 4 3}$ & $79.90 \%$ & 2.89 \\
\hline \multirow{2}{*}{3} & LR & $81.29 \%$ & 1.74 & $81.05 \%$ & 2.36 \\
& LIBSVM & $79.83 \%$ & 1.73 & $79.02 \%$ & 3.34 \\
\hline \multirow{2}{*}{3} & LIBSVM & $80.57 \%$ & 1.48 & $79.47 \%$ & 3.06 \\
& LR & $79.40 \%$ & 1.28 & $79.32 \%$ & 2.30 \\
\hline \multirow{2}{*}{2} & LR & $80.30 \%$ & 1.62 & $81.06 \%$ & 2.38 \\
& LIBSVM & $73.57 \%$ & 2.40 & $73.22 \%$ & 3.01 \\
\hline \multirow{2}{*}{2} & LR & $76.85 \%$ & 1.14 & $77.35 \%$ & 2.41 \\
& LIBSVM & $76.01 \%$ & 5.81 & $73.04 \%$ & 2.70 \\
\hline
\end{tabular}

5.81 while for LR it is rarely as high as 1.74 . In other words, SVM fits to data very well and even minor random changes of data causes instability of results accuracy. For this reason, when we execute our test procedure for certain model few times, we get results which differ even $2 \%$. According to our observations such an effect occurs mostly for a very high or very low parameter models. For logit regression effect has not been noticed, which may be encouraging to select that algorithm.

We also observed that for almost all cases the more data we exclude from the test results the worse accuracy and deviation we achieve (results with Jacknife exclusion of 10 samples are generally better), which is promising - it shows that overfitting does not occur and classifier is well generalizing to cases not known during learning. The exception from that rule are very high-deviation and some simple two-parameter models mentioned before. In such cases, there is a concern that because of its oversimplification or excessive complication hard learning takes place. For this reason, it seems more secure to use logit regression or four- to six-parameter model.

Analyzing sensitivity and specificity of the best results from Table II we have the following observations:

- for SVM differences between TPR or SPC and ACC are from $1.32 \%$ to $6.47 \%$, while for LR $1.06 \%$ to $7.8 \%$ which is quite similar,

- the exceptions are 2-parameter SVM models, for which this differences were up to $19.33 \%$,

- as mentioned before only five of all the examined SVM models achieved both parameters higher than $80 \%$, which is not a problem for many LR models - all five are presented in Table I.

We confirmed that one of the most important risk factors mentioned in literature [5], [6], [8], [9], [28], [29], [31] is the $G A G E$ which exists in almost all (all presented in Table I and II) of the models with acceptable accuracy. Most classifiers presented in the literature consist BWEIGHT and RES PIMV parameters. Unexpectedly, RESPIMV parameter is indeed present in SVM models in Table I with very good (over $82 \%$ of ACC) results, but it is not in any LR model worth to present. However, BWEIGHT-containing one was indeed on the third place with $82.67 \% A C C$, but it was the only one in the first twenty, which is a group with $A C C$ higher than $82 \%$. Among the most frequently mentioned parameters there is $\mathrm{FiO}_{2}$ which depends on the $\mathrm{AA}$ (Eq. 1) feature used in our work that is indeed present in the best model, but also only in five others of the best twenty. On the other hand, 


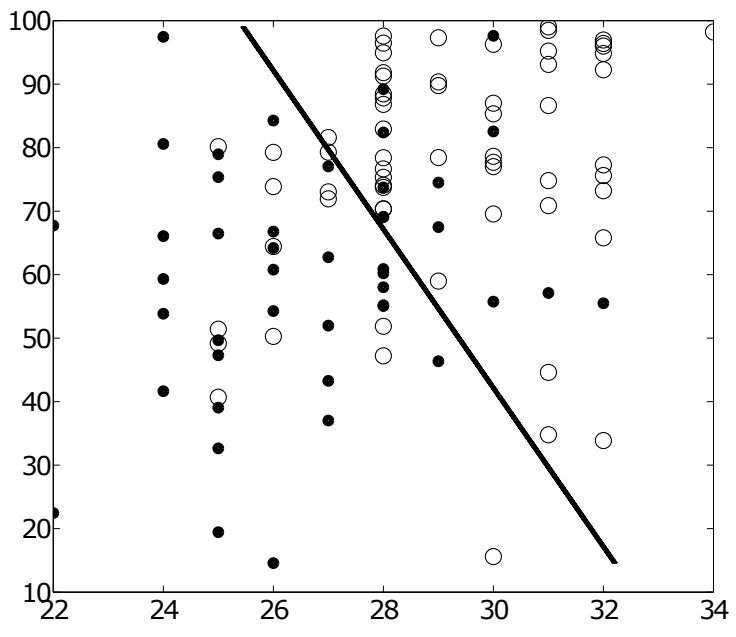

(a) Logistic regression

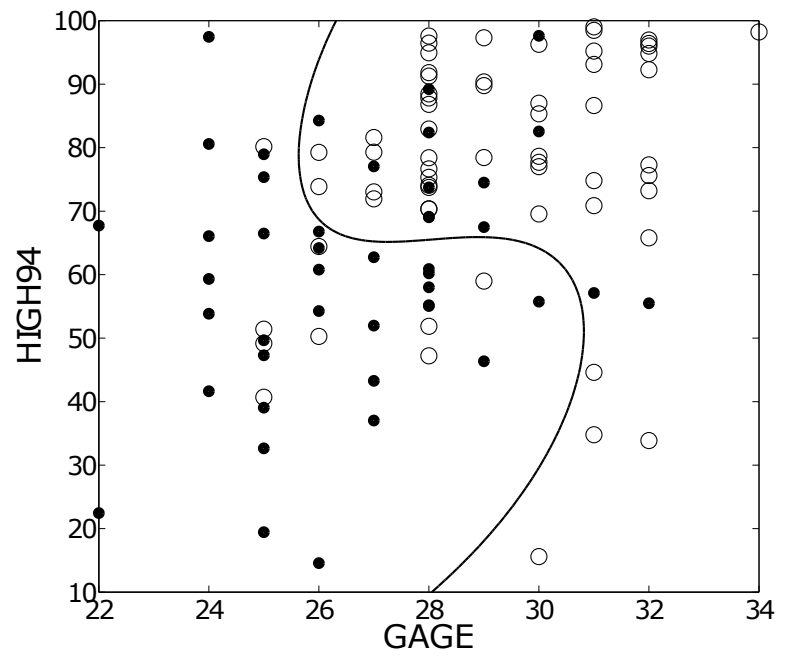

(b) Support Vector Machine

Fig. 4. Prediction result sample for two-parameter (GAGE, HIGH94).

$P D A$ feature appeared in 15 out of the best 20 models which is consistent with published results [8], [9], [25], [28]. The same situation occurs with $B P M M E A N$ parameter, which is the second most important factor after GAGE especially in LR models - it is in all of the first 20 models. The only one parameter related to $\mathrm{SpO}_{2}$ which is in more than half of the best 20 classifiers is LOW85. The average importance features are $S U R F A C T$ and HIGH94 while $S P O 2 D E V$, $S P O 2 D E V \_T R, S P O 2 M E A N \_T R$ parameters seem to have even less effect on the occurrence of diseases.

As a final conclusion we confirmed [32] that prediction of BPD after 7th day of life is possible with the accuracy higher than $82 \%$, not only with LR but also using Support Vector Machine algorithm. Results are slightly worse than in the Logit Regression method and more attention should be paid on model selection because many of them are sensitive to even small data changes. However, it can be very useful when we have limited set of parameters (above RESPIMV example), which are not so important in LR models as in SVM. Having wide scope of algorithms we can choose the one which is more suitable for our parameter set and thereby obtain better classification results. With that knowledge it is a good idea to construct expert system that would advise which algorithm and model to use having certain parameters measured.

\section{REFERENCES}

[1] Chih-Chung Chang and Chih-Jen Lin, LIBSVM : a library for support vector machines. ACM Transactions on Intelligent Systems and Technology, 2011, 2:27:1-27:27. Software available at http://www.csie.ntu.edu.tw/ cjlin/libsvm doi: 10.1145/1961189.1961199

[2] Ochab Marcin and Wiesław Wajs. Bronchopulmonary Dysplasia Prediction Using Support Vector Machine and Logit Regression. Information Technologies in Biomedicine, Volume 4. Springer International Publishing, 2014. 365-374. doi: 10.1007/978-3-319-06596-0_34
[3] Horbar JD, Badger GJ, Carpenter JH, Fanaroff AA, Kilpatrick S, LaCorte M, Phibbs R, Soll RF; Members of the Vermont Oxford Network. Trends in mortality and morbidity for very low birth weight infants, 1991-1999. Pediatrics 2002;110:143-151. doi: 10.1542/peds.110.1.143

[4] Stoll BJ, Hansen NI, Bell EF, Shankaran S, Laptook AR, Walsh MC, Hale EC, Newman NS, Schibler K, Carlo WA, et al.; Eunice Kennedy Shriver National Institute of Child Health and Human Development Neonatal Research Network. Neonatal outcomes of extremely preterm infants from the NICHD Neonatal Research Network. Pediatrics 2010;126:443-456. doi: 10.1542/peds.2009-2959

[5] Jobe, A. H.: The new bronchopulmonary dysplasia. Current opinion in pediatrics, 2011, 23(2), 167 doi: 10.1097/MOP.0b013e3283423e6b

[6] Groothuis, J. R., Makari, D.: Definition and outpatient management of the very low-birth-weight infant with bronchopulmonary dysplasia. Advances in therapy, 2012, 29(4), 297-311 doi: 10.1007/s12325-0120015-y

[7] Walsh, M. et al. Summary proceedings from the bronchopulmonary dysplasia group. Pediatrics 117(3), S52-56 (2006) doi: 10.1542/peds.20050620I

[8] Tapia, J.L., Agost, D., Alegria, A., Standen, J., Escobar, M., Grandi, C., et al.: Bronchopulmonary dysplasia: incidence, risk factors and resource utilization in a population of South American very low birth weight infants. Journal de Pediatria (Rio J). 2006, 82(1), 15-20 doi: 10.1590/S0021-75572006000100005

[9] Farstad, T., Bratlid, D., Medbø, S., Markestad, T.: Bronchopulmonary dysplasia-prevalence, severity and predictive factors in a national cohort of extremely premature infants. Acta Paediatrica, 2011, 100(1), pp. 5358 doi: 10.1111/j.1651-2227.2010.01959.x

[10] Ryan SW, Nycyk J, Shaw BN. Prediction of chronic neonatal lung disease on day 4 of life. Eur J Pediatr 1996;155:668-671. doi: 10.1007/BF01957150

[11] Subhedar NV, Hamdan AH, Ryan SW, Shaw NJ. Pulmonary artery pressure: early predictor of chronic lung disease in preterm infants. Arch Dis Child Fetal Neonatal Ed 1998;78:F20-F24. doi:10.1136/fn.78.1.F20

[12] Romagnoli C, Zecca E, Tortorolo L, Vento G, Tortorolo G. A scoring system to predict the evolution of respiratory distress syndrome into chronic lung disease in preterm infants. Intensive Care Med 1998;24: 476-480. doi: 10.1007/s001340050599

[13] Toce SS, Farrell PM, Leavitt LA, Samuels DP, Edwards DK. Clinical and roentgenographic scoring systems for assessing bronchopulmo- nary dysplasia. Am J Dis Child 1984;138:581— 585. doi:10.1001/archpedi.1984.02140440065017

[14] Corcoran JD, Patterson CC, Thomas PS, Halliday HL. Reduction in the risk of bronchopulmonary dysplasia from 1980-1990: results of a mul- 
tivariate logistic regression analysis. Eur J Pediatr 1993;152:677—681. doi: 10.1007/BF01955247

[15] Noack G, Mortensson W, Robertson B, Nilsson R. Correlations between radiological and cytological findings in early development of bron- chopulmonary dysplasia. Eur J Pediatr 1993;152:1024-1029. doi: 10.1007/BF01957230

[16] Yuksel B, Greenough A, Karani J. Prediction of chronic lung disease from the chest radiograph appearance at seven days of age. Acta Paediatr 1993;82:944-947. doi: 10.1111/j.1651-2227.1993.tb12605.x

[17] Bhutani VK, Abbasi S. Relative likelihood of bronchopulmonary dysplasia based on pulmonary mechanics measured in preterm neonates during the first week of life. J Pediatr 1992;120:605-613. doi: 10.1016/S00223476(05)82491-6

[18] Kim YD, Kim EA, Kim KS, Pi SY, Kang W. Scoring method for early prediction of neonatal chronic lung disease using modified respiratory parameters. J Korean Med Sci 2005;20:397-401. doi: 10.3346/jkms.2005.20.3.397

[19] Bhering CA, Mochdece CC, Moreira ME, Rocco JR, Sant'Anna GM. Bronchopulmonary dysplasia prediction model for 7-day-old infants. J Pediatr (Rio J) 2007;83:163-170. doi: 10.1590/S002175572007000200011

[20] Rojas MA, Gonzalez A, Bancalari E, Claure N, Poole C, Silva-Neto G. Changing trends in the epidemiology and pathogenesis of neonatal chronic lung disease. J Pediatr 1995;126:605—610. doi: 10.1016/S00223476(95)70362-4

[21] Marshall DD, Kotelchuck M, Young TE, Bose CL, Kruyer L, O'Shea TM. Risk factors for chronic lung disease in the surfactant era: a North Carolina population-based study of very low birth weight infants. North Carolina Neonatologists Association. Pediatrics 1999; 104:1345-1350. doi: $10.1542 /$ peds. 104.6.1345

[22] Oh W, Poindexter BB, Perritt R, Lemons JA, Bauer CR, Ehrenkranz RA, Stoll BJ, Poole K, Wright LL; Neonatal Research Network. Association between fluid intake and weight loss during the first ten days of life and risk of bronchopulmonary dysplasia in extremely low birth weight infants. J Pediatr 2005;147:786-790. doi: 10.1016/j.jpeds.2005.06.039
[23] Ambalavanan N, Van Meurs KP, Perritt R, Carlo WA, Ehrenkranz RA, Stevenson DK, Lemons JA, Poole WK, Higgins RD. NICHD Neo- natal Research Network, Bethesda, MD. Predictors of death or bronchopulmonary dysplasia in preterm infants with respiratory failure. J Perinatol 2008;28:420—426 doi: 10.1038/jp.2008.18

[24] Gilbert R., Keighley J., The arterial/alveolar oxygen tension ratio. An index of gas exchange applicable to varying inspired oxygen concentrations., Am Rev Respir Dis., 1974, 109, 142-145.

[25] Stoch, P.: Prediction of BronchoPulmonary Dysplasia in preterm neonates using statistical and artificial neural network tools. (Thesis or Dissertation style) Ph.D. dissertation, AGH University of Science and Technology, Kraków, 2007 (in Polish), pp. 60-72

[26] Kuenzel L. Predicting and undestanding bronchopulmonary dysplasia in permature infants. Stanford Undergraduate Research Journal

[27] Burges, C. J.C.: A Tutorial on Support Vector Machines for Pattern Recognition. Data Mining and Knowledge Discovery, 2, Kluwer Academic Publishers, Boston 1998, pp. 121-167 doi: 10.1023/A:1009715923555

[28] Sosenko, I. R., Bancalari, E.: New Developments in the Pathogenesis and Prevention of Bronchopulmonary Dysplasia. The Newborn Lung: Neonatology Questions and Controversies: Expert Consult-Online and Print, 2012, 217

[29] Cunha, G.S., Mezzacappa-Filho, F., Ribeiro, J. D.: Risk Factors for Bronchopulmonary Dysplasia in very Low Birth Weight Newborns Treated with Mechanical Ventilation in the First Week of Life. Journal of Tropical Pediatrics, 2005, 51(6), 334-340 doi: 10.1093/tropej/fmi051

[30] Jones, H. L.: Jacknife estimation of functions of stratum means. Biometrika, 1974, 61(2), 343-348 doi: 10.1093/biomet/61.2.343

[31] Ali, Z., Schmidt, P., Dodd, J., Jeppesen, D. L.: Bronchopulmonary dysplasia: a review. Archives of gynecology and obstetrics, 2013, 1-9 doi: 10.1007/s00404-013-2753-8

[32] Laughon, Matthew M., et al. "Prediction of bronchopulmonary dysplasia by postnatal age in extremely premature infants." American journal of respiratory and critical care medicine 183.12 (2011): 1715. doi: 10.1164/rccm.201101-0055OC 